\title{
7A52 Aluminum Alloy MIG Welding Residual Stress Reliable Measurement based on Hole-Drilling Method
}

\author{
Shiming Gan ${ }^{\mathrm{a}, \mathrm{b}}$, Yongquan Han ${ }^{\mathrm{b}, *}$, and Xiaoyan Bao ${ }^{\mathrm{a}, \mathrm{b}}$ \\ ${ }^{a}$ College of Mechanical Engineering, Inner Mongolia University of Technology, Hohhot, 010051, China \\ ${ }^{b}$ Materials Forming Key Laboratory, Inner Mongolia University of Technology, Hohhot, 010051, China
}

\begin{abstract}
To analyze the welding residual stress distributions for aluminum alloy medium and thick plates after the process of MIG welding, a residual stress testing system based on hole-drilling method was designed by virtual instrument and NI data acquisition card. To improve the accuracy and reliability of measurement results, the elasticity modulus error, strain gauge pasted error, and strain reading value time error were emphatically analyzed. The elasticity modulus error could be corrected by the curve that is fit to data measured in different MIG welding joint areas. The final measurement error caused by the strain gauge pasted error was reduced to 0 in 24 hours after the strain gauge was pasted. The final measurement error caused by the strain reading value time error was reduced to 0 in 150 minutes after the residual stress began to be measured. The experiment of MIG welding residual stress measurement was carried out on $10 \mathrm{~mm}$ thick 7A52 aluminum alloy plates. The results showed that the distributions of residual stresses on two sides of the weld seam were basically symmetrical about the weld center. The maximum tensile stress appeared in the fusion zone, and the maximum transverse residual stress $\sigma_{y}$ and the maximum longitudinal residual stress $\sigma_{x}$ were $96 \mathrm{MPa}$ and $185 \mathrm{MPa}$, respectively. The residual stresses from the fusion zone to heat affected zone were all tensile stresses, which were higher than the residual stresses in the center of the welding seam. Smaller compressive stresses appeared in the base metal.
\end{abstract}

Keywords: residual stress measurement; error analysis; metal inert gas welding; hole-drilling method

(Submitted on March 31, 2019; Revised on April 21, 2019; Accepted on June 20, 2019)

(C) 2019 Totem Publisher, Inc. All rights reserved.

\section{Introduction}

The 7A52 aluminum alloy is a kind of heat treatment strengthened aluminum alloy with high strength and good welding performance. It is the main welding structural material in the field of aerospace manufacturing and transportation. Among various welding methods of aluminum alloys, metal inert gas (MIG) welding has the advantages of easy automation and high production efficiency, and it has become the most widely used welding method for aluminum alloys [1-4]. MIG welding technology can achieve single side welding and double side forming for the $10 \mathrm{~mm}$ thick 7A52 aluminum alloy, and it greatly improves the welding production efficiency. However, in the process of MIG welding for aluminum alloy medium and thick plates, double layer welding is required, with a large heat input, small heating range, and large temperature gradient. At the same time, in order to reduce the impact of welding deformation on weld forming, constraints need to be imposed on the workpiece, resulting in large residual stress in the joint.

Welding residual stress has adverse effects on the fatigue strength, service life, stress corrosion, cracking resistance, and dimensional stability of welded structures, and it is also the main inducement of instability and cracking for welded structures in service. In order to ensure the reliability of welded structures, the distribution of residual stress after welding should be measured. In the existing residual stress measurement methods, the hole-drilling method is the most widely used residual stress measurement method with a complete theoretical system, and it was induced to the criteria of residual stress measurement by the American Society for Testing Material (ASTM). However, there are many error factors in the measurement of residual stress by the hole-drilling method, which affect the reliability of the measurement results. In order to reduce the influence of various error factors on the measurement results, it has been the focus to improve the reliability of

\footnotetext{
* Corresponding author.

E-mail address: 447172542@qq.com
} 
the measurement results of residual stress by the hole-drilling method. In references [5-12], the measurement errors of residual stress using the hole-drilling method caused by drilling eccentricity, deviation of pasting angle of strain gauge, hole edge plastic deformation, hole diameter and hole depth, testing surface shape, patch size, etc. were estimated and analyzed. To some extent, the reliability of residual stress measurement results under the common welding processes has been improved, but there are few reports on the MIG welding residual stress measurement and reliability analysis for aluminum alloy medium and thick plates using the hole-drilling method.

In this paper, a welding residual stress testing system based on the hole-drilling method was established. According to reference [13], the influences of the elasticity modulus error, strain gauge pasted error, and strain reading value time error on the measurement results were emphatically analyzed, and methods to eliminate these errors were given to ensure the reliability of the measurement results. The MIG welding residual stress measurement was carried out under specific process conditions, and the reliable distribution of residual stress was obtained. It may provide some necessary theoretical support for the regulation of MIG welding process parameters for aluminum alloy medium and thick plates.

\section{Residual Stress Measurement Method}

\subsection{Residual Stress Measurement Method}

Residual stress measurement methods are mainly divided into physical measurement methods and mechanical measurement methods [14-16], and the hole-drilling method is the most commonly used mechanical measurement method. The principle of the hole-drilling method is as follows. Referring to Figure 1, a small hole with radius a is drilled at the measured point. Since the original stress balance state is destroyed, slight deformation will occur around the small hole. The residual stres $s$ at the measured position can be obtained by computation according to the measured strain release, as shown in Equation (1).

$$
\left\{\begin{array}{l}
\sigma_{1,2}=\frac{1}{4 A}\left(\varepsilon_{0}+\varepsilon_{90}\right) \pm \frac{\sqrt{2}}{4 B} \sqrt{\left(\varepsilon_{0}-\varepsilon_{45}\right)^{2}+\left(\varepsilon_{90}-\varepsilon_{45}\right)^{2}} \\
\tan 2 \phi=\frac{\varepsilon_{0}+\varepsilon_{90}-2 \varepsilon_{45}}{\varepsilon_{0}-\varepsilon_{90}}
\end{array}\right.
$$

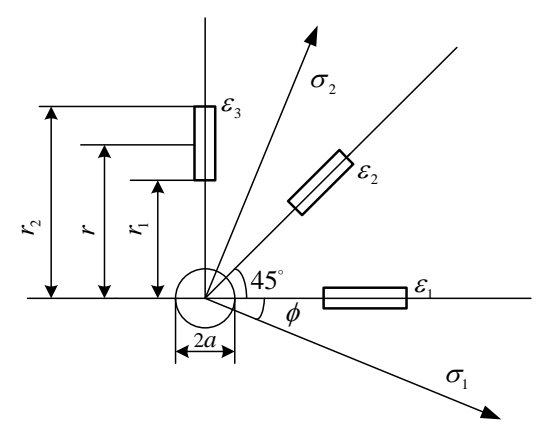

Figure 1. Schematic diagram of hole-drilling method

In Equation (1), $\varepsilon_{1}, \varepsilon_{2}$, and $\varepsilon_{3}$ are the strain release values measured by the strains of $0^{\circ}, 45^{\circ}$, and $90^{\circ}$, respectively. A and $\mathrm{B}$ are strain release coefficients. $\phi$ is the angle between the principal residual stress $\sigma_{1}$ and strain gauge in the direction of $0^{\circ}$ (the clockwise direction is positive). $\sigma_{1}$ and $\sigma_{2}$ are principal residual stresses.

\subsection{Residual Stress Testing System}

The hardware of the welding residual stress testing system consists of an industrial computer, sensor, and NI data acquisition card, as shown in Figure 2. The type of sensor is a resistance strain gauge with the BE120-2CA-K model. The data acquisition card is a model of the PXIe-4330 board card produced by the NI company. It has eight synchronous sampling analog input channels, and each has an anti-aliasing filtering function that can be calibrated independently. The sampling rate of each channel is $25 \mathrm{k} / \mathrm{s}$. A programmable $1 / 4$ bridge, half-bridge, and full-bridge resistance are built in each channel. The hole-drilling device consists of a positioning device, centering device, and electric drill with the J1Z-AN-6 model, and the rotation speed is $0-1400 \mathrm{r} / \mathrm{min}$. 


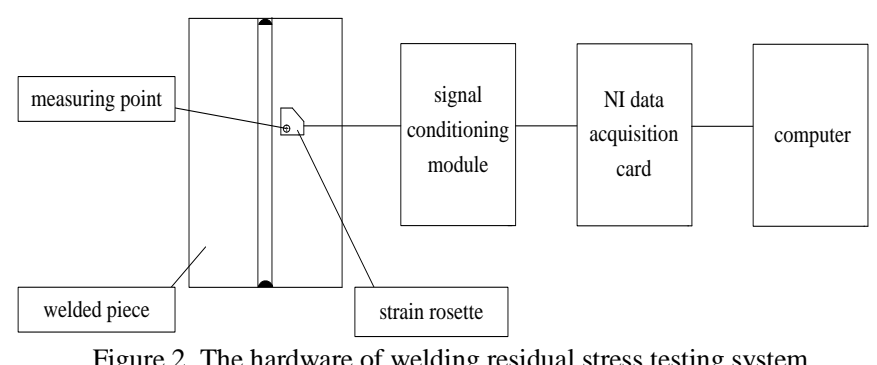

Figure 2. The hardware of welding residual stress testing system

The software of the welding residual stress testing system is based on the hole-drilling method and is written by the virtual instrument LabVIEW. It can collect, filter, display, and store the strain signal and complete the computation of transformation from strain to stress. The software system mainly consists of three parts: measuring the parameter record, measuring the module, and processing the data. The measuring module is the main part. It is divided into five sub-modules: the elasticity modulus measuring module, release coefficient calibration module, residual stress measuring module, data correction module, and error analysis module to realize reliable measurement for welding residual stress.

\section{Experimental Process}

In the experiment, the SUPER-MIG welding machine was used to carry out MIG double-layer butt welding on 7 A52 aluminum alloy plates with dimensions of $240 \mathrm{~mm}$ (length) $\times 100 \mathrm{~mm}$ (width) $\times 10 \mathrm{~mm}$ (thickness). The welding parameters that can obtain good weld forming were selected as follows. The groove angle was $60^{\circ}$, blunt edge was $2 \mathrm{~mm}$, and interpass temperature was $100^{\circ} \mathrm{C}$. The groove type of specimen is shown in Figure 3 . The current of the first layer was 201A, and the voltage was 20V. The current of the second layer was 309A, and the voltage was 22.9V. The welding speed was $400 \mathrm{~mm} / \mathrm{min}$. The flow of MIG protective gas was $13 \mathrm{~L} / \mathrm{min}$. In order to avoid large deformation after welding, four clamping bolts were used to fix the two sides of each aluminum alloy plate.

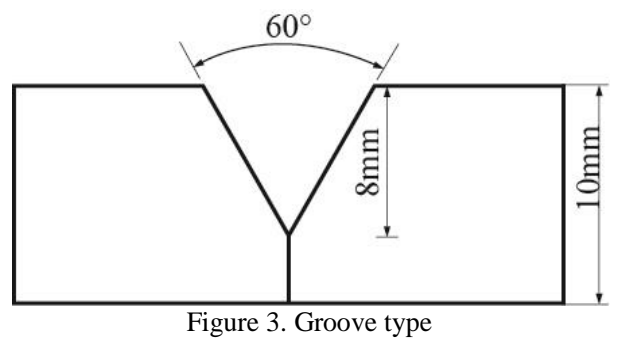

Based on the principle of the hole-drilling method, the residual stress measurement system proposed above was used to measure the residual stress of MIG welding. In the middle part of the weld length direction, the temperature fluctuation is small, and the welding parameters and the droplet transition are more stable, which makes the residual stress distribution in the middle part of the weld stable [17]. In order to measure the distribution of welding residual stress in different areas on both sides of the weld seam, the measuring point was taken at different positions in the middle of the testing piece perpendicular to the direction of the weld seam. In the experiment, the thermocouple was used to measure the surface temperature of the welding piece. The curves of the thermal cycle at different positions from the center of the weld seam were obtained. According to the curves of the thermal cycle, and because the temperature of the mechanical thermal melting point of the aluminum alloy material is about $500^{\circ} \mathrm{C}$, the positions of $0 \mathrm{~mm}, 6 \mathrm{~mm}, 20 \mathrm{~mm}$, and $45 \mathrm{~mm}$ from the center of the weld seam could be located in the weld zone, fusion zone, heat affected zone, and base metal zone, respectively. Therefore, in the direction perpendicular to the weld seam, section 1-1 was in the middle of the weld seam, section 2-2 was close to the position of arc starting, and section 3-3 was close to the position of the arc quenching. Sections 2-2 and 3-3 were both close to the bolt fixed area. On each section, five measuring points on the left and right sides of the weld seam were symmetrically taken and were $0 \mathrm{~mm}, 6 \mathrm{~mm}, 20 \mathrm{~mm}, 45 \mathrm{~mm}$, and60 $\mathrm{mm}$ from the center of the weld seam, as shown in Figure 4.

There are many factors influencing the measurement of welding residual stress by the hole-drilling method, and they often lead to large measurement errors that affect the reliability of measurement results. This indicates that there is insufficient data to evaluate the accuracy of the hole-drilling method in the standard of E837-81. In order to ensure the reliability of residual stress measurement results, based on the existing analysis for residual stress measurement error, this 
paper focuses on error analysis from the three aspects of elasticity modulus error, strain gauge pasted error, and strain reading value time error. The tensile specimens of 7A52 aluminum alloy MIG welding were prepared, and the strain gauge was pasted on the tensile specimens $2 \mathrm{~mm}, 13 \mathrm{~mm}, 20 \mathrm{~mm}$, and $40 \mathrm{~mm}$ away from the center of the weld seam, corresponding to the weld zone, the heat affected zone, the fusion zone, and the base metal zone, respectively. The actual elasticity modulus of each area was measured to analyze the impact of the elasticity modulus on the final measurement results. At the center of weld seam on section 1-1, the influence of the strain gauge pasted error and strain reading value time error on the final measurement result were both analyzed through the error transfer programs written by LabVIEW.

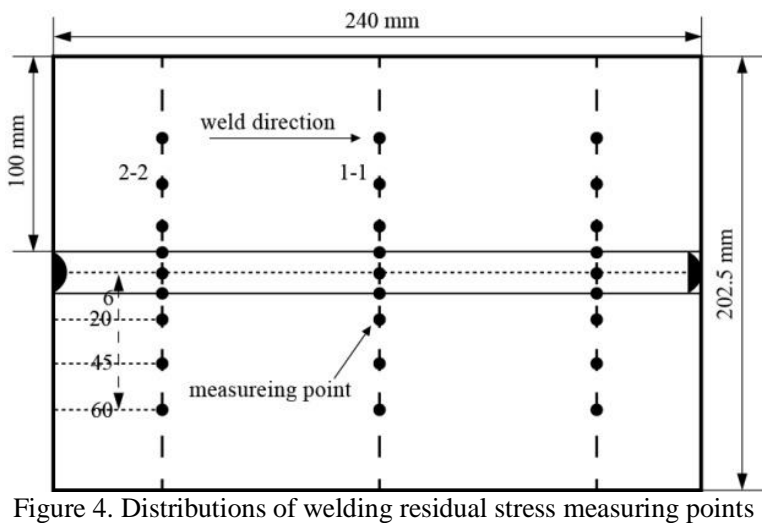

\section{Error Analysis}

\subsection{Analysis for Elasticity Modulus Error}

The elasticity modulus of the base metal of the 7A52 aluminum alloy is generally considered as 71GPa. According to Equation (1), the error between the measured elasticity modulus and the given value in each area after welding is indirectly transmitted to the final measurement result through the release coefficient. The measured elasticity modulus and relative error are shown in Table 1.

Table 1. Measured value of elasticity modulus

\begin{tabular}{|c|c|c|}
\hline Distance from weld center $(\mathrm{mm})$ & Elasticity modulus $(\mathrm{GPa})$ & Relative error \\
\hline 2 & 65 & $8.4 \%$ \\
\hline 13 & 66 & $7.0 \%$ \\
\hline 25 & 68.6 & $4.2 \%$ \\
\hline 40 & 70 & $1.4 \%$ \\
\hline
\end{tabular}

The data measured in Table 1 are all lower than the given value of $71 \mathrm{GPa}$. Therefore, when the given value is used to measure the residual stress in different areas on both sides of the weld seam, there will inevitably be an error. In order to eliminate the influence of the elasticity modulus error on the measurement results, the curve of elasticity modulus changing with the distance between the measuring point and the center of the weld was fitted according to the measured results in Table 1 to achieve the correction of elasticity modulus error, as shown in Figure 5.

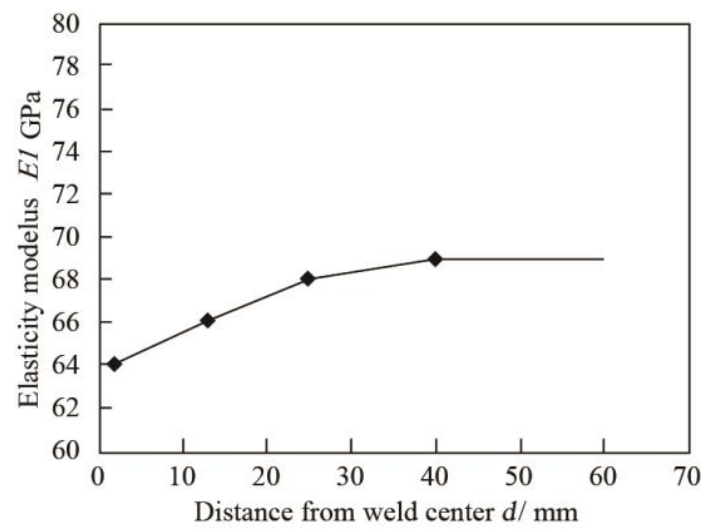

Figure 5. Fit curve of elasticity modulus 


\subsection{Error Analysis for Strain Gauge Pasted Error}

The strain gauge is pasted onto the specimen by means of instant solidification glue, such as 502 and manual pasting. Elastic shrinkage will inevitably occur in the process of glue bonding, which makes the strain gauge deform slightly. In the process of manual pasting, deformation of the strain gauge results and its resistance value changes, which generates the initial strain. These pre-formed strains can only be released within a certain period of time after the strain gauge is pasted. If the strain begins to be measured by drilling hole in a short time after the strain gauge is patched, strain errors will be inevitably produced, and then the residual principal stress $\sigma_{1}, \sigma_{2}$, and angle of $\phi$ will produce errors. In order to investigate the influence of the strain gauge pasted error on the final measurement results, while neglecting the influence of other error factors, a large number of measuring experiments without hole-drilling were carried out to record the strain values at different times under the same condition of the strain gauge being pasted. At the center position of the weld seam of the 7A52 aluminum alloy MIG welding specimen, the error transfer program was written by LabVIEW to analyze the relative errors of residual principal stress $\sigma_{1}, \sigma_{2}$, and angle of $\phi$ that were caused by the strain gauge pasted errors at different times, as shown in Figure 6.

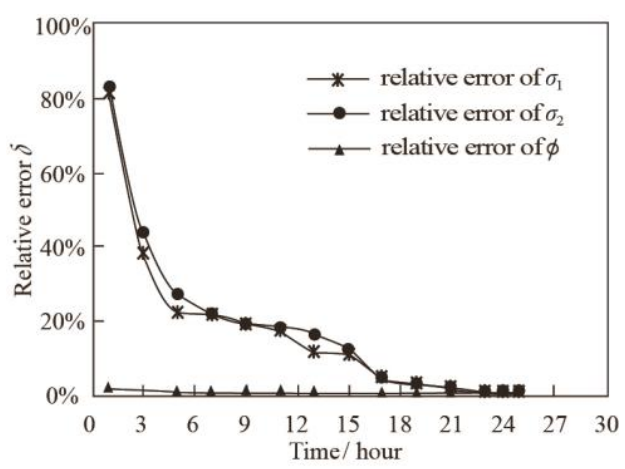

Figure 6 . The relative error curves caused by strain gauge pasted error

As can be seen from Figure 6, the residual principal stress $\sigma_{1}$ and $\sigma_{2}$ begin to be measured by the drilling hole in a short time after the strain gauge is pasted, and a large relative error is produced. The relative errors are gradually reduced as the strain release time increases, and the relative errors of the $\sigma_{1}, \sigma_{2}$, and $\phi$ angle are reduced to 0 after 24 hours, when the stress between the strain gauge and glue solidify layer is completely released. Therefore, the influence of the strain gauge pasted error on the measurement result of residual stress can be eliminated in 24 hours after the strain gauge is pasted.

\subsection{Analysis for Strain Reading Value Time Error}

After the strain gauge pasted error was eliminated, the strain release curve during the experiment was observed and analyzed, and it was found that it took a certain time for the strain release to reach the steady-state value. In the process of strain release, the strain value being read at any time produces a strain reading value time error, which could lead to final errors in the residual principal stress $\sigma_{1}, \sigma_{2}$, and angle of $\phi$ (neglecting the influence of other error factors). At the center position of the weld seam of the 7A52 aluminum alloy MIG welding specimen, the error transfer program was written by LabVIEW to analyze the relative error of residual principal stress $\sigma_{1}, \sigma_{2}$, and angle of $\phi$ that were caused by the strain reading value time error at different times, as shown in Figure 7.

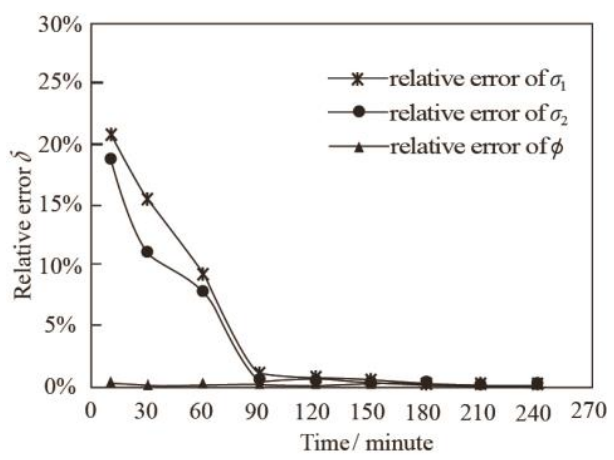

Figure 7. The relative error curve caused by strain reading value time error 
As shown in Figure 7, in the process of measuring residual stress by the drilling hole, the relative errors of the final principal residual stress $\sigma_{1}$ and $\sigma_{2}$ clearly demonstrate a decreasing trend within 90 minutes, but they change minimally after 90 minutes and decrease to 0 after 150 minutes. The relative error of the angle of $\phi$ is less than $0.1 \%$ in the whole measuring process, and it decreases to 0 after 150 minutes. Therefore, when the strain value is read for residual stress computation after 150 minutes of measurement, the influence of strain reading value time error on the final measurement results can be eliminated.

\section{Residual Stress Testing Results}

After the above measurement errors were corrected and eliminated, the welding residual stress testing system proposed in the paper was used to measure the transverse residual stress $\sigma_{y}$ and the longitudinal residual stress $\sigma_{x}$ for $10 \mathrm{~mm}$ thick 7A52 aluminum alloy plates after the process of MIG welding. The reliable results are shown in Figures 8 and 9.

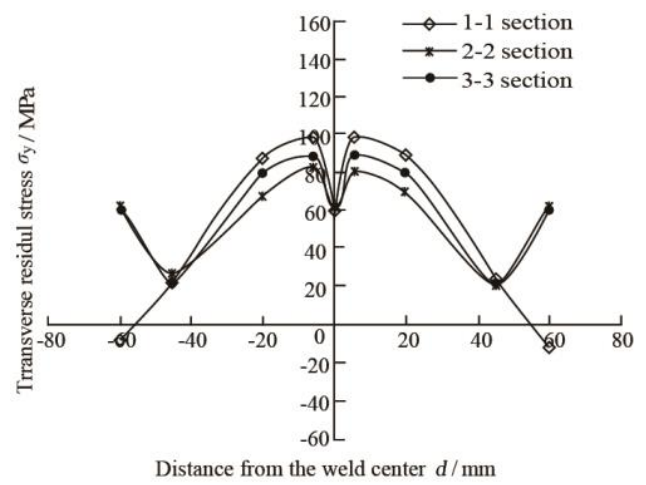

Figure 8. Transverse welding residual stress distributions

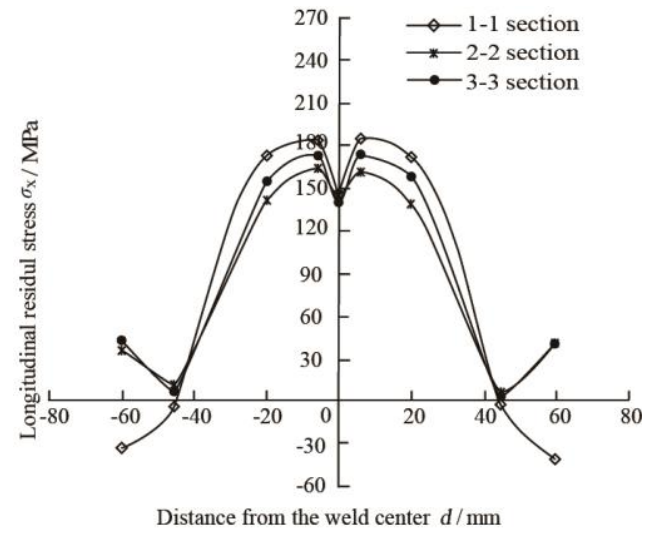

Figure 9. Longitudinal welding residual stress distributions

It can be seen from the measurement results in Figures 8 and 9 that the distributions of the transverse residual stress $\sigma_{y}$ and longitudinal residual stress $\sigma_{x}$ are similar for the three different measured sections of the 7A52 aluminum alloy plates after the process of MIG welding. The maximum tensile stress is $6 \mathrm{~mm}$ away from the center of the weld seam (the fusion zone), and the maximum transverse residual stress $\sigma_{y}$ and longitudinal residual stress $\sigma_{x}$ are $96 \mathrm{MPa}$ and $185 \mathrm{MPa}$, respectively. From the fusion zone to the heat affected zone, the transverse residual stress and the longitudinal residual stress are both tensile stress and higher than those in the weld zone. There is a small compressive stress in the base metal area, and the distributions of stress in each area on both sides of the weld seam are basically symmetrical about the weld center line. The reasons for the above characteristics of residual stress distribution are as follows. In the MIG welding process of aluminum alloy medium and thick plates, the overall heat input is large, and the heat is transferred from the center of the weld seam to both sides. Thus, the heat input from the fusion and heat affected zone is concentrated, and the temperature gradient is large. From the weld zone to the base metal zone, at the same measuring point from the weld center line, the residual stress values measured in section 1-1 are larger than those measured in the other two sections. However, at the measuring point $65 \mathrm{~mm}$ away from the weld center line in sections 2-2 and 3-3, due to their proximity to the clamped area, the transverse residual stress and longitudinal residual stress are much larger than the transverse residual stress and longitudinal residual stress measured in section 1-1.

\section{Conclusions}

A welding residual stress testing system based on the hole-drilling method was established by the LabVIEW virtual instrument and NI data acquisition card, and it can be used to measure the residual stress for aluminum alloy medium and thick plates after the process of MIG welding.

In order to ensure the reliability of measurement results, the elasticity modulus error, strain gauge pasted error, and strain reading value time error were all emphatically analyzed. Through the analysis of those errors, it was found that the elasticity modulus error can be corrected by the curve that is fit to data measured in different MIG welding joint areas. The relative error of the residual principal stress $\sigma_{1}, \sigma_{2}$, and $\phi$ angle caused by the strain gauge pasted error was reduced to 0 in 24 hours after the strain gauge was pasted. The relative error of the residual principal stress $\sigma_{1}, \sigma_{2}$, and $\phi$ angle caused by the strain reading value time error was reduced to 0 in 150 minutes after the residual stress began to be measured. 
For 10mm thick 7A52 aluminum alloy MIG welding plates, the maximum tensile stress appeared in the fusion zone, and the maximum transverse residual stress $\sigma_{y}$ and longitudinal residual stress $\sigma_{x}$ were $96 \mathrm{MPa}$ and $185 \mathrm{MPa}$, respectively. From the fusion zone to the heat affected zone, the transverse residual stress and the longitudinal residual stress were both tensile stress and higher than those in the center of the weld seam. The residual stress in the clamped area was obviously greater than that in the unclamped area.

\section{Acknowledgements}

This work is supported by the National Science Foundation of China (No. 51665044).

\section{References}

1. J. S. Thomsen, "Control of Pulsed Gas Metal Arc Welding," International Journal of Modelling, Identification and Control, Vol. 1, No. 2, pp. 115-125, 2006

2. X. W. Wang, Y. Huang, and Y. M. Zhang, "Droplet Transfer Model for Laser-Enhanced GMAW," International Journal of Advanced Manufacturing Technology, Vol. 64, No. 1-4, pp. 207-217, 2013

3. H. Terasaki and S. W. Simpson, "Modeling of the GMAW System in Free Flight and Short Circuiting Transfer," Science and Technology of Welding and Joining, Vol. 10, No. 1, pp. 120-124, 2005

4. S. Krishnan, D. V. Kulkarni, and A. De, "Multipass Pulsed Current Gas Metal Arc Welding of P91 Steel," Sci. Technol. Weld. Joining, Vol. 21, No. 3, pp. 171-177, 2016

5. X. P. Zhang and N. Wang, "Measurement of Welding Residual Stress in The Heavy Converter using Blind-Hole Method," Mechanics in Engineering, Vol. 29, No. 3, pp. 45-49, 2007

6. M. Barsanti, M. Beghini, L. Bertini, B. Monelli, and C. Santus, "First-Order Correction to Counter the Effect of Eccentricity on the Hole-Drilling Integral Method with Strain-Gage Rosettes," Journal of Strain Analysis Engineering Design, Vol. 51, No. 6, pp. 431-443, 2016

7. K. Zhang, M. Yuan, and J. Chen, "General Calibration Formulas for Incremental Hole Drilling Optical Measurement," Experimental Techniques, Vol. 41, No. 1, pp. 1-8, 2017

8. C. Sergey, K. Nikolai, K. Benjamin, and H. Norbert, "Artificial Neural Network for Correction of Effects of Plasticity in Equibiaxial Residual Stress Profiles Measured by Hole Drilling," Journal of Strain Analysis for Engineering Design, Vol. 52, No. 3, pp. 137-151, 2017

9. R. Richter and T. Müller, "Measurement of Residual Stresses: Determination of Measurement Uncertainty of the Hole-Drilling Method used in Aluminum Alloys," Experimental Techniques, Vol. 41, No. 1, pp. 79-85, 2017

10. H. D. Wang, Y. Zhou, K. Shi, and Y. M. Liu, "Sticking Technology of Resistance Strain Gauge in Determining Test of Residual Stress by The Blind-Hole Technique," Welded Pipe and Tube, Vol. 32, No. 3, pp. 35-37, 2009

11. H. Y. Zhao, Y. Pei, Y. W. Shi, and H. Wang, "The Influence and Correction to the Measuring Error of High Welding Residual Stress when using Hole-Drilling Method," Journal of Mechanical Strength, Vol. 18, No. 3, pp. 17-20, 1996

12. L. Wang and L. Y. Xie, "The Influence of Friction Stir Welding Process for Materials Elastic Modulus," Welding Technology, Vol. 38, No. 8, pp. 22-25, 2009

13. S. M. Gan, Y. Q. Han, and F. R. Chen, "Analysis on Error Factors of Welding Residual Stress Measured by Hole Drilling Method," Transactions of the China Welding Institution, Vol. 39, No. 10, pp. 48-53, 2018

14. N. J. Rendler and I. Vigness, "Hole-Drilling Strain-Gage Method of Measuring Residual Stresses," Experimental Mechanics, Vol. 6, No. 12, pp. 577-586, 1966

15. R. A. Owen, R. V. Preston, P. J. Withers, H. R. Shercliff, and P. J. Webster, "Neutron and Synchrotron Measurements of Residual Strain in TIG Welded Aluminum Alloy 2024," Materials Science and Engineering, Vol. 24, No. 346, pp. 159-167, 2003

16. G. Albertini, G. Bruno, B. D. Dunn, et al., "Comparative Hole-Drilling and X-Ray Residual Stress Measurements on Steel Welded Plate," Materials Science and Engineering, No. 224, pp. 157-162, 1997

17. C. Q. Huang, H. Li, C. G. Luo, and Y. L. Song, "Comparative Study of Blind Hole Method and Indentation Method in Measuring Residual Stress of 2219 Aluminium Alloy Arc-Welded Joint," Transactions of the China Welding Institution, Vol. 38, No. 7, pp. 54-38, 2017

Shiming Gan is a doctoral candidate at Inner Mongolia University of Technology. His research interests include welding residual stress tests.

Yongquan Han is a professor at Inner Mongolia University of Technology. His research interests include new welding processes and automation.

Xiaoyan Bao is a doctoral candidate at Inner Mongolia University of Technology. Her research interests include welding heat source simulation. 